\title{
PREVALÊNCIA DE DOENÇAS CRÔNICAS NÃO TRANSMISSÍVEIS, HÁBITOS ALIMENTARES E DE ATIVIDADE FÍSICA NUMA ESTRATÉGIA DE SAÚDE DA FAMÍLIA DE PRESIDENTE PRUDENTE - SP
}

\author{
PREVALENCE OF CHRONIC NON-COMMUNICABLE DISEASES, EATING HABITS \\ AND PHYSICAL ACTIVITY IN A FAMILY HEALTH STRATEGY IN PRESIDENTE \\ PRUDENTE - SP
}

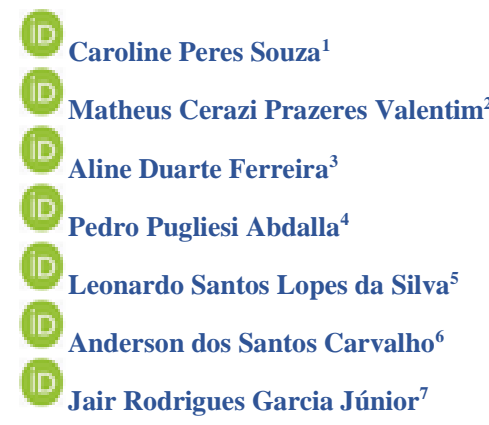

Avaliado e aprovado pelo Comitê de Ética em Pesquisa (CAAE n.12209619.8.0000.5515)

Autor correspondente

Jair Rodrigues Garcia Júnior

Curso de Educação Física - UNOESTE - campus II

Rod. Raposo Tavares, Km 572, Limoeiro.

CEP 19067-175, Presidente Prudente, SP

+55 (18) 99151-8542

jgjunior@unoeste.br

${ }^{1}$ Graduada em Educação Física, Universidade do Oeste Paulista (UNOESTE), Presidente Prudente, SP, Brasil.

carol_peres10@hotmail.com

${ }^{2}$ Graduado em Educação Física, Universidade do Oeste Paulista (UNOESTE), Presidente Prudente, SP, Brasil.

matheus_cerazi@hotmail.com

${ }^{3}$ Curso de Fisioterapia, Universidade do Oeste Paulista (UNOESTE), Presidente Prudente, SP, Brasil.

aline@unoeste.br

${ }^{4}$ Doutorando em Ciências - Escola de Enfermagem de Ribeirão Preto, Universidade de São Paulo (USP), Ribeirão Preto, SP, Brasil.

pedroabdalla11@gmail.com

${ }^{5}$ Graduando em Educação Física - Universidade Paulista (UNIP), São José do Rio Preto, SP, Brasil.

leonsilva_unip@outlook.com

${ }^{6}$ Doutor em Ciências - Curso de Educação Física, Universidade Paulista (UNIP), São José do Rio Preto, SP, Brasil

ander_uai@hotmail.com

${ }^{7}$ Doutor em Ciências - Curso de Educação Física, Universidade do Oeste Paulista (UNOESTE), Presidente Prudente, SP, Brasil

\section{Cite como}

Vancouver

Souza, CP, Valentim, MCP, Ferreira, AD, Abdalla, PP, Silva, LSL, Carvalho, AS. et al. Prevalência de doenças crônicas não transmissíveis, hábitos alimentares e de atividade física numa estratégia de saúde da família de Presidente Prudente - SP. Conscientiae Saúde 2020;19(1):1-15, e18221. https://doi.org/10.5585/conssaude.v19n1.18221.

\section{Resumo}

Introdução: As doenças crônicas não transmissíveis (DCNT) têm elevada prevalência na população brasileira e atingem todas as classes sociais, representando aproximadamente $71 \%$ das mortes no mundo.

Objetivo: Investigar a prevalência de pacientes com DCNT registradas em um posto da Estratégia de Saúde da Família (ESF) em Presidente Prudente e verificar os hábitos alimentares e a prática de atividade física.

Métodos: A pesquisa foi baseada em informações de prontuários $(n=1005)$ e questionários (hábitos alimentares e práticas de atividade física). A amostra foi composta por maiores de 18 anos de uma ESF de Presidente Prudente-SP.

Resultados: A maior prevalência foi de hipertensão arterial, principalmente, em pacientes acima de 80 anos de idade. Metade da população estudada não tem hábitos alimentares adequados e apenas um terço dos adultos tem prática suficiente de atividade física.

Conclusão: Hipertensão arterial (30\%), obesidade (17\%) e Diabetes Mellitus (9\%) são as DCNT mais prevalentes. A redução da prevalência futura das DCNT dependerá da forma como educaremos as gerações subsequentes.

Descritores: Adulto. Exercício Físico. Posto de Saúde. Sistema Único de Saúde.

Abstract

Introduction: Noncommunicable diseases (NCDs) have a high prevalence in the Brazilian population and affect all social classes, representing about $71 \%$ of deaths worldwide.

Objective: to investigate the prevalence of patients with NCDs registered at a Family Health Strategy (FHS) post in Presidente Prudente and to verify eating habits and physical activity.

Methods: The research was based on information from medical records $(n=1005)$ and questionnaires (eating habits and physical activity practices). The sample consisted of people over 18 years old from an FHS in Presidente Prudente-SP.

Results: The highest prevalence was arterial hypertension, mainly in patients over 80 years of age. Half of the population studied does not have adequate eating habits and only a third of adults have sufficient physical activity.

Conclusion: Arterial hypertension (30\%), obesity (17\%) and Diabetes Mellitus $(9 \%)$ are the most prevalent NCDs. The reduction in the future prevalence of NCDs will depend on how we educate subsequent generations.

Keywords: Adult. Physical Exercise. Health Center. Unified Health System. 
Introdução

As doenças crônicas não transmissíveis (DCNTs) são doenças que se instalam no organismo de maneira silenciosa e gradativa, podendo demorar anos até que se manifestem sinais e sintomas ${ }^{1,2}$. As DCNTs têm consequências para o indivíduo, para sua família, para o sistema público de saúde e até para a economia. Podem causar o empobrecimento das famílias, em razão dos custos para o tratamento contínuo, pois em sua maioria não tem cura, causam limitações para a empregabilidade e a manutenção da renda. Para o sistema público de saúde, o ônus é contínuo e crescente, o que acaba por enfraquecer a economia do país. A elevada prevalência dessas doenças está frustrando os objetivos de redução de pobreza nos países em desenvolvimento, pois o direcionamento de recursos para o tratamento reduz os investimentos que poderiam ser alocadas no desenvolvimento social e econômico ${ }^{3}$.

De acordo com a Organização Mundial de Saúde (OMS) ${ }^{4}$, no mundo, as DCNTs são responsáveis por $71 \%$ das mortes (41 milhões de pessoas) por ano. As principais DCNTs causadoras dessas mortes são: doenças cardiovasculares (17,9 milhões), câncer (9 milhões), doenças respiratórias (3,9 milhões) e diabetes (1,6 milhões). A $\mathrm{OMS}^{3}$ tem uma projeção na qual o número de mortes por DCNTs terá aumento significativo de $15 \%$ entre 2010 e 2020, representando aproximadamente 44 milhões de mortes. A maior proporção de mortes está prevista para os países em desenvolvimento e de baixa renda, localizados na África, Sudeste Asiático e Mediterrâneo Oriental, onde o aumento estimado é de mais de $20 \%$.

No Brasil, foi criada a Atenção Primária à Saúde (APS) com o intuito de promover cuidados de saúde para a população. A APS é definida por um conjunto de ações de saúde, no âmbito pessoal e público, incluindo a promoção, a proteção e a manutenção da saúde, a prevenção de agravos, o diagnóstico, o tratamento e a reabilitação. É ampliada por meio de práticas gerenciais e sanitárias democráticas e participativas, incluindo trabalho em equipe, dirigidas a populações de regiões bem delimitadas, para as quais se atribui a responsabilidade sanitária, considerando a dinamicidade existente na região em que habitam esses públicos. Também são empregadas tecnologias de elevada complexidade, porém de limitada abrangência, pois a prioridade é a resolução de problemas de saúde de maior regularidade e relevância em cada região. Por isso, o contato prioritário dos usuários com os sistemas de saúde se caracteriza pelos princípios da universalidade, da acessibilidade e da coordenação do cuidado, do vínculo e da continuidade, da integralidade, da responsabilidade, da humanização, da equidade e da participação social ${ }^{5}$. 
A APS conceitua o sujeito em sua singularidade, na complexidade, na integralidade, na inserção sociocultural e procura a promoção de sua saúde, a prevenção e tratamento de doenças e a redução de danos ou de sofrimentos que sejam capazes de comprometer sua possibilidade de viver de modo saudável ${ }^{5}$.

A Estratégia de Saúde da Família (ESF) representa a reorganização da APS no País, conforme as normas do Sistema Único de Saúde (SUS) ${ }^{5}$. A ESF tem caráter substitutivo à rede de APS tradicional (centralizada em hospitais). Por isso, nas regiões em que as ESFs atuam há expansão das atividades conforme o diagnóstico situacional, o planejamento de ações e a programação da execução. O foco primário é a família e a comunidade, caracterizando um espaço de idealização da cidadania e alguns outros princípios, além de atender aos princípios gerais da $\mathrm{APS}^{5}$.

As DCNTs são as maiores causas de morte no mundo, podendo apresentar grande variação das prevalências específicas de acordo com as regiões em um município. Dessa forma, o estabelecimento dessas prevalências pode representar uma informação importante para que a Secretaria de Saúde e outras autoridades direcionem melhor as estratégias, os recursos humanos e financeiros. Nesse sentido, este estudo teve como objetivo investigar a prevalência de pacientes com DCNTs registradas em um posto da ESF de Presidente Prudente-SP e verificar os hábitos alimentares e a prática de atividade física.

\section{Material e métodos}

Esta pesquisa foi de caráter retrospectivo e prospectivo, descritiva, com abordagem quantitativa baseada em coleta de informações em prontuários e por meio de questionários. Foi avaliada e aprovada pelo Comitê de Ética em Pesquisa (CAAE n. 12209619.8.0000.5515).

Participaram da pesquisa indivíduos de ambos os sexos, cadastrados e inscritos no posto da ESF de dois bairros do município de Presidente Prudente-SP, que se enquadraram nos seguintes critérios de inclusão: indivíduos com idade $\geq 18$ anos e com prontuários arquivados na ESF. Como critério de exclusão: pacientes com registro de óbito. Houve autorização prévia do Secretário Municipal de Saúde para a realização da coleta de dados nos prontuários.

Foi solicitada dispensa da assinatura do Termo de Consentimento Livre e Esclarecido (TCLE) para aqueles participantes $(96,4 \%$ ) que tiveram os dados coletados exclusivamente em seus prontuários arquivados no posto da ESF. Para os participantes que tiveram os dados coletados nos prontuários e também por meio dos questionários (3,6\%) (análise prospectiva), houve um convite para participação voluntária quando de sua visita rotineira na própria ESF. 
Estes foram esclarecidos sobre os objetivos e procedimentos da pesquisa e, aqueles que concordaram, assinaram o TCLE.

Para a análise retrospectiva foram coletadas informações de 1.005 prontuários. Os dados registrados para análise posterior foram o número do prontuário, sexo, idade, se era tabagista, etilista, peso, estatura, doenças crônicas diagnosticadas e tempo de diagnóstico. O peso e a estatura foram utilizados para calcular o Índice de Massa Corporal (IMC) em $\mathrm{kg} / \mathrm{m}^{2}$ e foi classificado o estado nutricional em baixo peso, peso normal, sobrepeso e obesidade ${ }^{6}$.

Para a análise prospectiva, foram aplicados dois questionários em 71 pacientes durante sua visita rotineira ao posto ESF. O primeiro continha perguntas sobre hábitos alimentares, conforme proposto e validado por Gomes et al. ${ }^{7}$. O segundo questionário continha perguntas sobre a prática habitual de atividades físicas, conforme proposto por Baecke et al ${ }^{8}$, traduzido e validado por Florindo e Latorre ${ }^{9}$. A prática habitual de atividades físicas compreende três domínios: a) ocupacional, b) exercício físico no lazer; c) lazer e locomoção. Para atribuir pesos a cada um dos domínios e classificar a prática habitual atividade física a partir do escore total, foi utilizada a classificação proposta pelos mesmos autores: fraco $(2$ a 3,9), regular $(4$ a 5,9) e bom $(6$ a 7,9$)$.

Foi utilizada a estatística descritiva para apresentação dos dados em frequências absoluta e relativa, considerando o sexo e as faixas etárias $(18+, 40+, 60+$ e 80+).

\section{Resultados}

Foram analisados 1.005 prontuários, que incluíam o total de 1.969 pacientes, divididos em dois bairros: no Bairro 1 havia 626 homens e 840 mulheres e no Bairro 2 havia 198 homens e 305 mulheres. As doenças que apresentaram maior prevalência em homens e mulheres e em ambos os bairros foram hipertensão arterial com 30\% e Diabetes Mellitus com 9\% (Tabela 1 e Figura 1). Na maioria das doenças, quanto maior foi a faixa etária, maior foi a prevalência das mesmas; exceto para as dislipidemias que tiveram predomínio nos 40+ (Bairro 1 e homens do Bairro 2) e nos 60+ para mulheres do Bairro 2 (Tabela 1 e Figura 1). 
Tabela 1 - Prevalência (frequências absoluta e relativa) de doenças crônicas por Bairro, sexo e faixa etária

\begin{tabular}{|c|c|c|c|c|c|c|c|c|c|}
\hline & & $\begin{array}{l}\text { Faixa etária } \\
\quad \text { (anos) }\end{array}$ & $\mathbf{N}$ & $\begin{array}{c}\text { Diabetes } \\
\text { n }(\%)\end{array}$ & $\begin{array}{l}\text { H. Arterial } \\
\text { n (\%) }\end{array}$ & $\begin{array}{c}\text { Dislipidemias } \\
\text { n }(\%)\end{array}$ & $\begin{array}{l}\text { D. Card.* } \\
\text { n (\%) }\end{array}$ & $\begin{array}{c}\text { D. } \\
\text { Respirat. } \\
\text { n (\%) }\end{array}$ & $\begin{array}{l}\text { D. Sist. } \\
\text { Nerv. } \\
\text { n (\%) }\end{array}$ \\
\hline \multirow{10}{*}{ •ֶ, } & \multirow{5}{*}{ 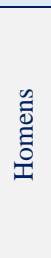 } & $80+$ & 38 & $10(26)$ & $23(61)$ & $2(5)$ & $4(11)$ & $2(5)$ & 0 \\
\hline & & $60+$ & 159 & $41(26)$ & $95(60)$ & $7(4)$ & $7(4)$ & $5(3)$ & $5(3)$ \\
\hline & & $40+$ & 197 & $26(13)$ & $61(31)$ & $12(6)$ & $7(4)$ & $5(3)$ & $2(1)$ \\
\hline & & $18+$ & 232 & $3(1)$ & $16(7)$ & $4(2)$ & 0 & 0 & 0 \\
\hline & & Total & 626 & $80(13)$ & $195(31)$ & $25(4)$ & $18(3)$ & $12(2)$ & $7(1)$ \\
\hline & \multirow{5}{*}{ 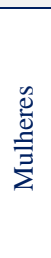 } & $80+$ & 69 & $13(19)$ & $50(72)$ & $1(1)$ & $3(4)$ & $3(4)$ & $3(4)$ \\
\hline & & $60+$ & 226 & $43(19)$ & $126(56)$ & $17(8)$ & $11(5)$ & $7(3)$ & $2(1)$ \\
\hline & & $40+$ & 281 & $22(8)$ & $84(30)$ & $28(10)$ & $5(2)$ & $5(2)$ & $1(0,4)$ \\
\hline & & $18+$ & 264 & $3(1)$ & $8(3)$ & $2(1)$ & $5(1)$ & $3(1)$ & 0 \\
\hline & & Total & 840 & $81(7)$ & $268(32)$ & $48(6)$ & $24(3)$ & $18(2)$ & $6(0,7)$ \\
\hline \multirow{10}{*}{ 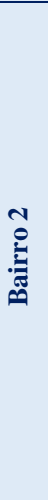 } & \multirow{5}{*}{ 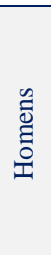 } & $80+$ & 8 & $1(13)$ & $5(63)$ & 0 & $2(25)$ & 0 & $1(13)$ \\
\hline & & $60+$ & 57 & $8(14)$ & $23(40)$ & $2(4)$ & $1(2)$ & $1(2)$ & 0 \\
\hline & & $40+$ & 63 & $10(16)$ & $21(33)$ & $6(10)$ & 0 & $1(2)$ & $1(2)$ \\
\hline & & $18+$ & 70 & $2(3)$ & $5(7)$ & 0 & 0 & 0 & $1(1)$ \\
\hline & & Total & 198 & $21(11)$ & $54(27)$ & $8(4)$ & $3(1)$ & $2(1)$ & $3(1)$ \\
\hline & \multirow{5}{*}{ 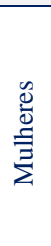 } & $80+$ & 20 & $6(30)$ & $12(60)$ & $2(10)$ & $1(5)$ & 0 & 0 \\
\hline & & $60+$ & 73 & $15(21)$ & $35(48)$ & $10(14)$ & $3(4)$ & $3(4)$ & 0 \\
\hline & & $40+$ & 109 & $7(6)$ & $26(24)$ & $9(8)$ & $2(2)$ & $1(1)$ & $1(1)$ \\
\hline & & $18+$ & 104 & 0 & $5(5)$ & $2(2)$ & 0 & $1(1)$ & $1(1)$ \\
\hline & & Total & 306 & 28 (9) & $78(25)$ & $23(7)$ & $6(2)$ & $5(2)$ & $2(1)$ \\
\hline
\end{tabular}

*: Infarto do miocárdio, acidente vascular cerebral (AVC), angina, endocardite, arritmias, dentre outras.

Fonte: Autores. 
Figura 1 - Frequência relativa da prevalência de doenças crônicas no Bairro 1 de homens (A) e mulheres (B) e Bairro 2 de homens (C) e mulheres (D)

$100 \%$ (A) Homens do Bairro 1

(B) Mulheres do Bairro 1

$\square 18+\square 0+\square 60+\square 80+\square$ Total

$50 \%$

$\%$

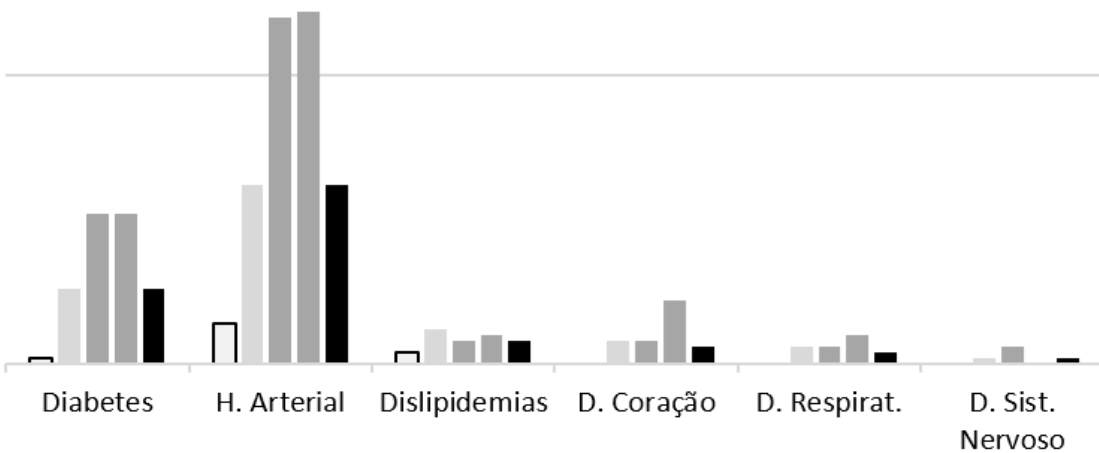

(C) Homens do Bairro 2

$50 \%$

$0 \%$

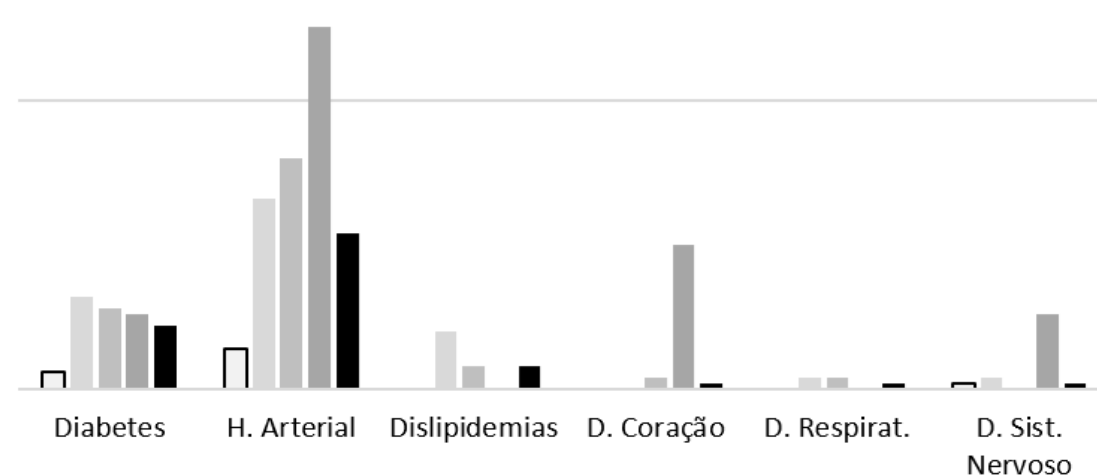

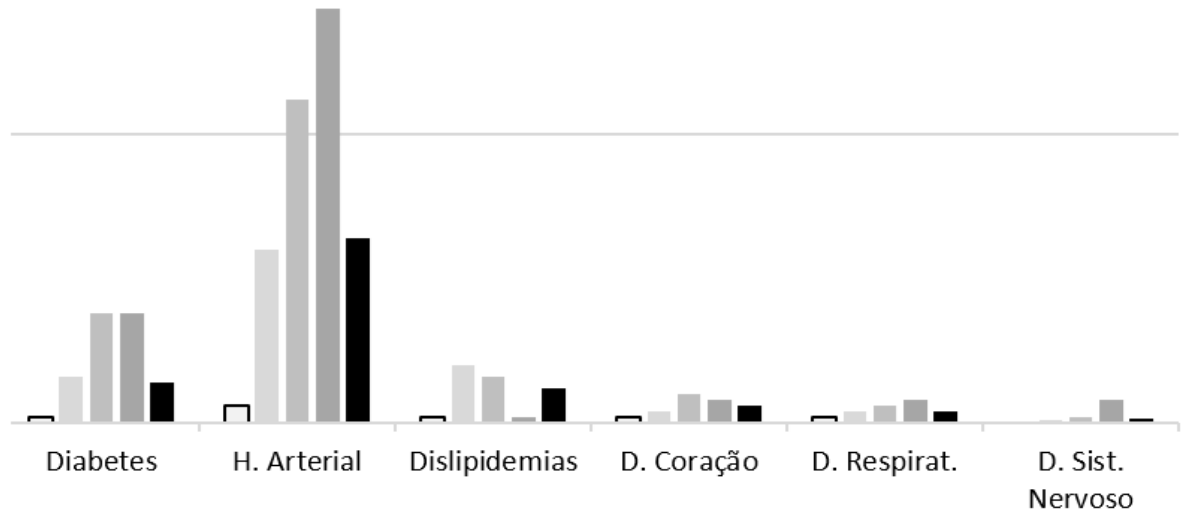

(D) Mulheres do Bairro 2

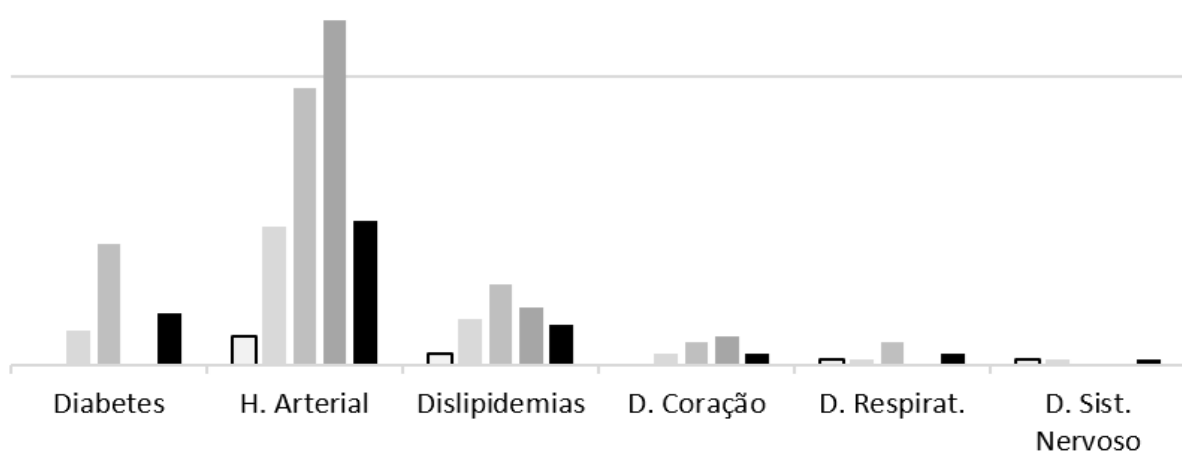

Fonte: Autores 
Sobre o estado nutricional dos participantes nos dois bairros, observamos que o sobrepeso e até a obesidade foram mais prevalentes que o peso normal (Figura 2 e Tabela 2). Nos homens de ambos os bairros, o sobrepeso foi o estado nutricional mais prevalente. Nas mulheres de ambos os bairros, a obesidade foi o estado nutricional mais prevalente. Observa-se tendência de queda para obesidade à medida que a idade cronológica é maior.

Tabela 2 - Frequências absoluta e relativa do estado nutricional classificado por meio do Índice de Massa Corporal (IMC) por bairro, sexo e faixa etária

\begin{tabular}{|c|c|c|c|c|c|c|c|c|}
\hline & & \multirow[b]{2}{*}{ Faixa etária (anos) } & \multirow[b]{2}{*}{$\mathbf{n}$} & \multirow[b]{2}{*}{ n com IMC } & \multicolumn{4}{|c|}{ Estado nutricional } \\
\hline & & & & & $\begin{array}{c}\text { Baixo peso } \\
\text { n }(\%)\end{array}$ & $\begin{array}{c}\text { Normal } \\
\text { n }(\%)\end{array}$ & $\begin{array}{c}\text { Sobrepeso } \\
\text { n }(\%)\end{array}$ & $\begin{array}{c}\text { Obesidade } \\
\text { n }(\%)\end{array}$ \\
\hline \multirow{10}{*}{ 苞 } & \multirow{5}{*}{ 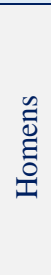 } & $80+$ & 38 & 18 & $2(11)$ & $4(22)$ & $10(56)$ & $2(11)$ \\
\hline & & $60+$ & 159 & 65 & $0(0)$ & $22(34)$ & $33(51)$ & $10(15)$ \\
\hline & & $40+$ & 197 & 78 & $1(1)$ & $25(32)$ & $22(28)$ & $30(38)$ \\
\hline & & $18+$ & 232 & 78 & $1(1)$ & $29(37)$ & $30(38)$ & $18(23)$ \\
\hline & & Total & 626 & 239 & $4(2)$ & $80(33)$ & $95(40)$ & $60(25)$ \\
\hline & \multirow{5}{*}{$\frac{\mathscr{d}}{\stackrel{0}{0}}$} & $80+$ & 69 & 25 & $1(4 \%)$ & $9(36)$ & $10(40)$ & $5(20)$ \\
\hline & & $60+$ & 226 & 93 & $0(0)$ & $30(32)$ & $32(34)$ & $31(33)$ \\
\hline & & $40+$ & 281 & 143 & $0(0)$ & $24(17)$ & $52(36)$ & $67(47)$ \\
\hline & & $18+$ & 264 & 118 & $4(3)$ & $47(40)$ & $36(31)$ & $31(26)$ \\
\hline & & Total & 840 & 379 & $5(1)$ & $110(29)$ & $130(34)$ & $134(35)$ \\
\hline \multirow{10}{*}{ ְֶ. } & \multirow{5}{*}{$\begin{array}{l}\text { D } \\
\text { ป̃ } \\
\text { I }\end{array}$} & $80+$ & 8 & 5 & $1(20)$ & $2(40)$ & $1(20)$ & $1(20)$ \\
\hline & & $60+$ & 57 & 44 & $1(2)$ & $14(32)$ & $18(41)$ & $11(25)$ \\
\hline & & $40+$ & 63 & 45 & $0(0)$ & $9(20)$ & $20(44)$ & $16(36)$ \\
\hline & & $18+$ & 70 & 43 & $4(9)$ & $16(37)$ & $11(26)$ & $12(28)$ \\
\hline & & Total & 198 & 137 & $6(4)$ & $41(30)$ & $60(44)$ & $50(36)$ \\
\hline & \multirow{5}{*}{ 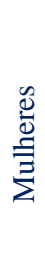 } & $80+$ & 20 & 12 & $1(8)$ & $2(17)$ & $9(75)$ & $0(0)$ \\
\hline & & $60+$ & 73 & 55 & $1(2)$ & $12(22)$ & $19(35)$ & $23(42)$ \\
\hline & & $40+$ & 109 & 76 & $1(1)$ & $16(21)$ & $16(21)$ & $43(57)$ \\
\hline & & $18+$ & 104 & 67 & $5(7)$ & $24(36)$ & $19(36)$ & $19(28)$ \\
\hline & & Total & 306 & 210 & $8(4)$ & $54(26)$ & $63(30)$ & $85(40)$ \\
\hline
\end{tabular}

Fonte: Autores. 
Figura 2 - Frequência relativa do estado nutricional no Bairro 1 de homens (A) e mulheres (B) e Bairro 2 de homens (C) e mulheres (D)

$100 \%$ (A) Homens do Bairro 1

$50 \%$

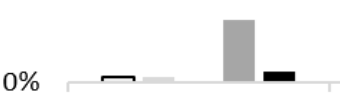

Baixo peso

$100 \%$

(C) Homens do Bairro 2
(B) Mulheres do Bairro 1

$$
\square 18+\square 40+\square 60+\square 80+\square \text { Total }
$$

$50 \%$

Fonte: Autore

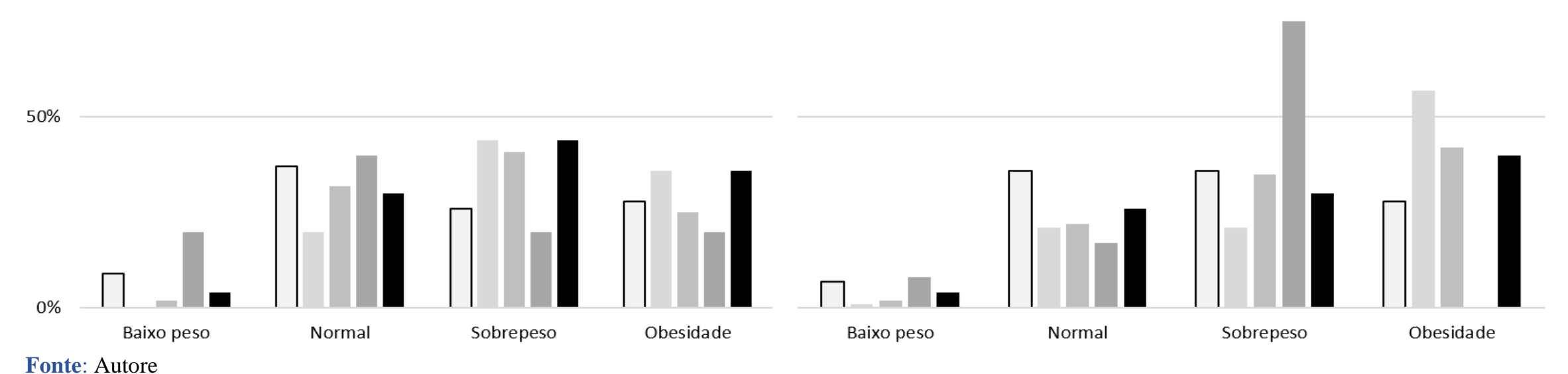


Sobre os hábitos alimentares e a prática de atividades físicas, o número de questionários respondidos foi de 71, sendo 17 de homens e 54 de mulheres. Dentre os respondentes homens, mais da metade (53\%) eram etilistas e $12 \%$ eram tabagistas. Dentre as mulheres, $13 \%$ eram etilistas e $7 \%$ eram tabagistas.

Sobre os hábitos alimentares, dos homens que consomem margarina, nenhum opta pela variedade light. Entre as mulheres que consomem margarina, apenas $7 \%$ opta pela variedade light. Em geral, pôde ser percebido que a proporção de hábitos alimentares saudáveis é maior do que dos hábitos inadequados (Tabela 3). A exceção fica com o consumo de refrigerante elevado para as mulheres $(70 \%)$ e o consumo para algumas vezes na semana de frituras, biscoito recheado e bolinhos doces, pois somente $35 \%$ dos homens e $26 \%$ das mulheres não realizam esse consumo frequente.

Tabela 3 - Consumo alimentar em frequência relativa por sexo

\begin{tabular}{|c|c|c|}
\hline Itens & Homens (\%) & Mulheres (\%) \\
\hline \multicolumn{3}{|l|}{ Consumo de manteiga e margarina } \\
\hline Sim para manteiga & 41 & 37 \\
\hline Sim para margarina & 24 & 30 \\
\hline Não consomem nenhuma delas & 29 & 17 \\
\hline \multicolumn{3}{|l|}{ Consumo de laticínios } \\
\hline Sim para leite integral & 51 & 69 \\
\hline Não consomem leite & 22 & 15 \\
\hline Sim para iogurte, queijo e requeijão tradicional/integral & 63 & 87 \\
\hline Não consomem iogurte, queijo e requeijão & 33 & 6 \\
\hline \multicolumn{3}{|l|}{ Consumo de refrigerantes e sucos } \\
\hline Não para refrigerante & 47 & 30 \\
\hline Sim para refrigerante com açúcar & 35 & 52 \\
\hline Sim para suco de fruta natural & 41 & 63 \\
\hline Sim para suco em pó & 29 & 33 \\
\hline Sim para suco de polpa de fruta & 18 & 13 \\
\hline \multicolumn{3}{|l|}{ Consumo de frituras } \\
\hline Sim para todos os dias & 29 & 15 \\
\hline Sim para algumas vezes na semana & 65 & 74 \\
\hline \multicolumn{3}{|l|}{ Consumo de biscoitos recheados e bolinhos doces } \\
\hline Sim para todos os dias & 35 & 19 \\
\hline Sim para algumas vezes na semana & 65 & 74 \\
\hline \multicolumn{3}{|l|}{ Consumo de verduras, legumes e frutas } \\
\hline Sim para verduras e legumes todos os dias & 65 & 72 \\
\hline Sim para frutas todos os dias & 47 & 52 \\
\hline
\end{tabular}

Fonte: Autores. 
A avaliação da prática habitual de atividade física teve escores que variaram de 3,75 até 7,75 para os homens e de 2,75 até 8,0 para as mulheres. A classificação dos escores da prática habitual de atividade física proposta por Valério ${ }^{10}$ revelou que para a maior parte dos participantes foi "regular", tanto em homens como em mulheres, seguidas da classificação "boa" e com menor prevalência a "fraco" (Tabela 4).

Tabela 4 - Classificação, frequências absolutas e relativas dos escores da prática habitual de atividade física de acordo com os tercis em homens e mulheres

\begin{tabular}{|c|c|c|c|c|}
\hline \multirow{2}{*}{ Tercis } & \multirow{2}{*}{ Valores dos escores } & \multirow{2}{*}{ Classificação } & \multirow{2}{*}{$\begin{array}{c}\text { Homens } \\
\text { N (\%) }\end{array}$} & \multirow{2}{*}{$\begin{array}{c}\text { Mulheres } \\
\mathbf{N}(\%)\end{array}$} \\
\hline & & & & \\
\hline 3 & 6 a 8 & Bom & $6(35,3)$ & $17(31,5)$ \\
\hline 2 & 4 a 5,9 & Regular & $10(58,8)$ & $26(48,1)$ \\
\hline 1 & 2 a 3,9 & Fraco & $1(5,9)$ & $11(20,4)$ \\
\hline
\end{tabular}

Fonte: Autores.

\section{Discussão}

O objetivo da pesquisa foi investigar a prevalência de pacientes com doenças crônicas registradas em um posto da Estratégia de Saúde da Família (ESF) de Presidente Prudente, além de verificar seus hábitos alimentares e de prática habitual de atividade física.

Analisando o Bairro 1, tanto em homens como em mulheres, foi constatada maior prevalência de hipertensão arterial, principalmente, na faixa etária a partir dos 80 anos. Uma das razões para esse resultado pode ser a elevada prevalência de pacientes com sobrepeso e obesidade, que são fatores de risco significativos para o desenvolvimento da hipertensão arterial. Além disso, o envelhecimento também é um fator de risco para a incidência da doença ${ }^{11}$. No Bairro 2, foi constatada situação semelhante, com prevalência maior de hipertensão arterial entre todas as demais doenças analisadas, principalmente, na faixa etária a partir dos 80 anos. Quando comparado ao IMC, encontra-se também maior prevalência de sobrepesados e obesos em ambos os sexos. A hipertensão arterial é a doença crônica mais popular observada pelos médicos ${ }^{12}$. Dados do Vigitel $^{13}$, durante doze anos (2006 a 2017), indicam que a prevalência de hipertensão arterial nos indivíduos adultos, residentes nas capitais, diferenciou de $23 \%$ a $24,3 \%$ respectivamente, sem mudanças em todo o período analisado, inclusive por sexo. No ano de 2017, com dados consolidados, as proporções entre adultos com 18 a 34 anos, o índice foi de 13,8\%; de 35 a 54 anos foi de 47,4\%; de 55 a 64 anos foi de 46,5\%; e de 65 anos ou mais, foi de $60,9 \%$. Logo, quando comparado ao estudo, encontramos dados similares.

Segundo a $\mathrm{OMS}^{6}$, em 2016, a porcentagem de adultos com mais de 18 anos e com excesso de peso era maior que $39 \%$ da população, e indivíduos obesos chegavam a $13 \%$ da população. Quando comparado a nossa pesquisa, vemos dados parecidos somente com o sobrepeso, já a obesidade atinge 
de $25-40 \%$ dos pacientes analisados em ambos os sexos, demonstrando proporções maiores quando comparados à população mundial. Segundo a $\mathrm{OMS}^{6}$, a obesidade é definida como acúmulo incomum ou exagerado de gorduras, que leva ao desenvolvimento de prejuízos à saúde do indivíduo. A obesidade é considerada uma patologia multifatorial, pois resulta de vários fatores que atuam de forma separada ou simultânea: genéticos, endócrinas, ambientais, culturais, socioeconômicos, psicológicos. Entre os fatores concorrentes para o disparo e desenvolvimento da obesidade, o que prevalece na maioria dos casos é o consumo alimentar desequilibrado com superávit calórico diário em conjunto com estilo de vida sedentário ${ }^{1,2}$. O fato ocorrido em nosso estudo da prevalência da obesidade e do valor médio do IMC serem menores com o envelhecimento é esperado. O peso corporal tende a diminuir nos idosos devido à doença da sarcopenia, caracterizada pela redução da força e massa muscular ${ }^{14}$ que predispõe idosos ao aumento de riscos à sua saúde, como quedas, hospitalização, perda da independência física e morte prematura ${ }^{15}$. Desse modo, em idosos passa a ser essencial considerar a composição corporal para avaliar a quantidade de gordura e o estado nutricional $^{16}$.

Com a terceira maior prevalência de DCNTs em nossa amostra, observamos o Diabetes Mellitus, em ambos os bairros e sexos. A razão para essa predominância pode ser a elevada prevalência de pacientes com os fatores de risco, tais como sobrepeso, obesidade, hábitos alimentares inadequados e sedentarismo ${ }^{17}$. O Diabetes Mellitus se refere a uma doença metabólica caracterizada por hiperglicemia decorrente de defeitos na ação da insulina, da secreção de insulina ou ambos. A hiperglicemia crônica do diabetes está relacionada a danos em longo prazo, como disfunção e insuficiência de diferentes órgãos, principalmente, olhos, rins, nervos, coração e vasos sanguíneos ${ }^{18}$. Além de a doença em si ser prejudicial, o diabetes tem várias consequências relacionadas com maiores taxas de hospitalização, maiores necessidades de cuidados médicos, maior incidência de doenças cardiovasculares e cerebrovasculares, cegueira, insuficiência renal e amputações não traumáticas de membros inferiores. Desse modo, há grande ônus para os sistemas de saúde dos países latino-americanos, onde ainda há dificuldade no controle de doenças infecciosas ${ }^{19}$. O Diabetes Mellitus tipo 2 corresponde a 90-95\% das pessoas com diabetes, referido também como diabetes adulto. A princípio, a vida destes pacientes é longa, não sendo necessário o tratamento com insulina para sobrevivência, mas apenas medicamentos específicos. As causas mais comuns para esse tipo de diabetes são sobrepeso, obesidade, sedentarismo e inflamação sistêmica crônica de grau elevado, juntamente com pré-disposição genética ${ }^{18}$. O Diabetes Mellitus tipo 1 representa apenas 5-10\% das pessoas com diabetes, também denominado pelos termos diabetes insulino-dependente ou diabetes de início juvenil, sendo consequência da destruição autoimune das células Beta pancreáticas mediada por leucócitos ${ }^{18}$. Em nosso estudo, não foi diferenciado os tipos de diabetes. 
Em relação aos hábitos alimentares, em ambos os sexos, observa-se baixa proporção de consumo de alimentos light/diet. Como houve uma prevalência considerável de diabéticos, é preciso conscientizar as pessoas com essa doença da importância de realizar trocas de alguns alimentos tradicionais por essas opções. A maioria dos homens (67\%) não consome produtos derivados do leite por fermentação (iogurte, queijo e requeijão), e esse público precisa ser conscientizado sobre a importância desses alimentos. Uma vez que o consumo dos derivados fermentados do leite pode reduzir o risco de câncer colorretal, Diabetes Mellitus tipo 2, facilitar a manutenção do peso corporal e melhorar a saúde cardiovascular, óssea e gastrointestinal, além de prevenir o câncer de mama nas mulheres ${ }^{20}$.

Outro fator merecedor de atenção é o consumo elevado de refrigerantes no público feminino. Os profissionais da ESF poderiam realizar orientações a respeito da importância da redução do consumo dessas bebidas ${ }^{21}$ e também incentivar essas mulheres para substituírem a bebida por água saborizada com frutas, ou raízes e folhas de sabor "refrescante" como o gengibre e a hortelã. Para ambos os sexos, é preciso incentivar a redução do consumo frequente de frituras, pois possuem maior densidade calórica e podem contribuir para a incidência do sobrepeso e obesidade. Além disso, podem aumentar o risco para problemas cardiovasculares e câncer de pulmão pelo modo de preparo desses alimentos envolverem a formação da acroleína, um componente derivado do glicerol dos triglicerídeos presentes no óleo da fritura ${ }^{22}$. O consumo frequente de biscoito recheado relatado é outro problema, pois esses alimentos são ricos em gorduras, incluindo algumas vezes a gordura trans, que aumentam as chances de doenças coronarianas ${ }^{23}$. Os pontos positivos foram que a maior proporção da amostra opta por alimentos com pouco açúcar e/ou gorduras e também foi observado que a maioria dos respondentes consome vegetais diariamente ou com frequência, o que é um fator primário para a prevenção de DCNTs, inclusive para diversos tipos de câncer ${ }^{24}$.

Para a prática de atividades físicas, a maior predominância para ambos os sexos foi no nível regular, porém, as mulheres apresentaram frequência de 20,4\% no nível fraco e os homens ficaram com pouco menos de $6 \%$ neste nível. Assim, é preciso estimular os frequentadores dessa ESF, principalmente as mulheres, para melhorarem sua classificação da prática habitual de atividade física. No âmbito do SUS, a maior parte das ações realizadas pelos profissionais de Educação Física é voltada para a população idosa, englobando pessoas pertencentes ao nível secundário de atenção à saúde $^{25}$. Assim, como há prevalência elevada de DCNTs também nos adultos (18+ e 40+), são necessárias mais ações para esse público. Também é preciso maior investimento e atenção dos profissionais de Educação Física no nível da APS, uma vez que é possível conscientizar a população e prevenir DCNTs futuras. Nesse sentido, foi publicado no Diário Oficial da União a resolução ${ }^{\circ}$ 391, de 26 de agosto de 2020, do Conselho Federal de Educação Física, a qual reconhece a atuação 
dos Profissionais de Educação Física no contexto hospitalar no âmbito do SUS em todos os níveis de atenção à saúde ${ }^{26}$. Assim, os reflexos esperados desse reconhecimento são o aumento do número e da qualidade das ações realizadas pelos profissionais do movimento no âmbito da APS, o que pode contribuir para melhor qualidade de vida da população.

\section{Conclusão}

Concluímos que as DCNTs atingem proporção elevada da população da ESF de Presidente Prudente e as mais comuns são a hipertensão arterial, obesidade e Diabetes Mellitus. Ao mesmo tempo, cerca da metade deles não pratica hábitos alimentares adequados e apenas um terço dos adultos tem prática suficiente de atividades físicas. Neste estudo, foram coletadas as informações de apenas um posto da ESF que atende a dois bairros, o que nos leva a sugerir que outros postos da cidade também sejam objeto de levantamento semelhante. São necessárias mais ações e estímulo dos profissionais da saúde para alertar e conscientizar os frequentadores dessa ESF quanto a seus hábitos alimentares e de atividade física, principalmente, pela influência que exercem sobre seus filhos. A redução da prevalência futura das DCNTs dependerá da forma como educaremos as gerações subsequentes.

\section{Agradecimentos}

Enfermeira responsável pela Estratégia de Saúde da Família Vivian Trondoli Nascimento, agradecemos pelo auxílio na localização e compreensão dos prontuários.

\section{Referências}

1. Silva LS, Cotta RMM, Rosa CdOB. Estratégias de promoção da saúde e prevenção primária para enfrentamento das doenças crônicas: revisão sistemática. Rev Panam Salud Publica.

2013;34(5):343-50.

2. Malta DC, Gosch CS, Buss P, Rocha DG, Rezende R, Freitas PC, et al. Doenças Crônicas Não Transmissíveis e o suporte das ações intersetoriais no seu enfrentamento. Cien Saude Colet. 2014;19(11):4341-50.

3. OMS. Global status report on noncommunicable diseases. 2010. [cited 202019 Sep]. Available from: https://www.who.int/nmh/publications/ncd_report2010/en/.

4. OMS. Noncommunicable diseases. 2010. [cited 202019 Sep]. Available from: https://www.who.int/news-room/fact-sheets/detail/noncommunicable-diseases. 
5. BRASIL. Ministério da Saúde. Secretaria de Atenção à Saúde. Departamento de Atenção Básica. Política nacional de atenção básica. 2012. [cited 202023 Jan]. Available from: http://bvsms.saude.gov.br/bvs/publicacoes/politica_nacional_atencao_basica_4ed.pdf.

6. OMS. Appropriate body-mass index for Asian populations and its implications for policy and intervention strategies. Lancet (London, England). 2004;363(9403):157-63.

7. Gomes AA, Pereira RA, Massae Yokoo EM. Caracterização do consumo alimentar de adultos por meio de questionário simplificado: contribuição para os estudos de vigilância alimentar e nutricional. Cad Saude Colet. 2015;23(4).

8. Baecke JA, Burema J, Frijters JE. A short questionnaire for the measurement of habitual physical activity in epidemiological studies. Amer J Clin Nutr. 1982;36(5):936-42.

9. Florindo A, Latorre M. Validação e reprodutibilidade do questionário de Baecke de avaliação da atividade física habitual em homens adultos. Rev Bras Med Esporte. 2003;9(3):121-8.

10. Valério DF. Correlação do consumo máximo de oxigênio com os índices de aptidão física obtidos nos questionários IPAQ e BAECKE [Trabalho de Conclusão de Curso (graduação)]. Biblioteca Digital da UNICAMP: UNICAMP; 2011.

11. Chen SC, Lo TC, Chang JH, Kuo HW. Variations in aging, gender, menopause, and obesity and their effects on hypertension in taiwan. Int J Hypertens. 2014;2014:515297.

12. Hart JT, Savage W. Tudo Sobre Hipertensão Arterial. São Paulo: Editora Andrei; 2016.

13. BRASIL. Ministério da Saúde. Secretaria de Vigilância em Saúde Departamento de Vigilância de Doenças e Agravos não Transmissíveis e Promoção da Saúde. Vigitel Brasil 2017: vigilância de fatores de risco e proteção para doenças crônicas por inquérito telefônico: estimativas sobre frequência e distribuição sociodemográfica de fatores de risco e proteção para doenças crônicas nas capitais dos 26 estados brasileiros e no Distrito Federal em 2017. 2018.

14. Abdalla PP, Carvalho AS, Santos AP, Venturini ACR, Alves TC, Mota J, et al. Cut-off points of knee extension strength allometrically adjusted to identify sarcopenia risk in older adults: A crosssectional study. Arch Gerontol Geriatr. 2020;89:104100.

15. Abdalla PP, Carvalho AS, Santos AP, Venturini ACR, Alves TC, Mota J, et al. One-repetition submaximal protocol to measure knee extensor muscle strength among older adults with and without sarcopenia: a validation study. BMC Sports Sci Med Rehabil. 2020;12(1):29.

16. Carvalho AS, Alves TC, Abdalla PP, Venturini ACR, Leites PDL, Machado DRL. Composição corporal funcional: breve revisão. Cad Ed Fís Esp. 2018;16(1):1-12.

17. Guariguata L, Brown C, Sobers N, Hambleton I, Samuels TA, Unwin N. An updated systematic review and meta-analysis on the social determinants of diabetes and related risk factors in the Caribbean. Rev Panam Salud Publica. 2018;42:e171.

18. American Diabetes Association. Diagnosis and Classification of Diabetes Mellitus. Diabetes Care. 2014;37(Supplement 1):S81. 
19. Sartorelli DS, Franco LJ. Tendências do diabetes mellitus no Brasil: o papel da transição nutricional. Cad Saude Publica. 2003;19:S29-S36.

20. Savaiano DA, Hutkins RW. Yogurt, cultured fermented milk, and health: a systematic review. Nutr Rev. 2020.

21. Vargas-Garcia EJ, Evans CEL, Prestwich A, Sykes-Muskett BJ, Hooson J, Cade JE. Interventions to reduce consumption of sugar-sweetened beverages or increase water intake: evidence from a systematic review and meta-analysis. Obes Rev. 2017;18(11):1350-63.

22. Henning RJ, Johnson GT, Coyle JP, Harbison RD. Acrolein Can Cause Cardiovascular Disease: A Review. Card Tox. 2017;17(3):227-36.

23. Wanders AJ, Zock PL, Brouwer IA. Trans Fat Intake and Its Dietary Sources in General Populations Worldwide: A Systematic Review. Nutrients. 2017;9(8).

24. Hurtado-Barroso S, Trius-Soler M, Lamuela-Raventós RM, Zamora-Ros R. Vegetable and Fruit Consumption and Prognosis Among Cancer Survivors: A Systematic Review and Meta-Analysis of Cohort Studies. Adv Nutr (Bethesda, Md). 2020.

25. Carvalho AS, Abdalla PP, Bueno Júnior CR. Atuação do profissional de educação física no sistema único de saúde: revisão sistemática. Rev Bras Prom Saúde. 2017;30(3).

26. CONSELHO FEDERAL DE EDUCAÇÃO FÍSICA. Resolução No 391, de 26 de Agosto de 2020. 2020. [cited 202019 Sep]. Available from Diário Oficial da União (DOU): https://ilape.edu.br/download/confef-resolucao-no-391-de-26-de-agosto-de-2020/\#. 